\title{
Reactions of nitroxides 15. Cinnamates bearing a nitroxyl moiety synthesized using a Mizoroki-Heck cross-coupling reaction
}

\author{
Jerzy Zakrzewski and Bogumiła Huras
}

\author{
Full Research Paper \\ Address: \\ Institute of Industrial Organic Chemistry, Annopol 6, 03-236 Warsaw, \\ Poland \\ Email: \\ Jerzy Zakrzewski* - zakrzewski@ipo.waw.pl \\ * Corresponding author \\ Keywords: \\ 4-acryloyloxy-2,2,6,6-tetramethylpiperidine-1-oxyl; cinnamates; \\ Mizoroki-Heck cross-coupling reaction; nitroxides
}

\author{
Beilstein J. Org. Chem. 2015, 11, 1155-1162. \\ doi:10.3762/bjoc. 11.130 \\ Received: 01 April 2015 \\ Accepted: 25 June 2015 \\ Published: 13 July 2015 \\ For part XIV see [1]. \\ Associate Editor: J. A. Murphy \\ (c) 2015 Zakrzewski and Huras; licensee Beilstein-Institut. \\ License and terms: see end of document.
}

\begin{abstract}
Cinnamic acid derivatives bearing a nitroxyl moiety (2,2,6,6-tetramethyl-1-oxyl-4-piperidyl 3-E-aryl acrylates) were synthesized in 30-100\% yield using a Mizoroki-Heck cross-coupling reaction between 4-acryloyloxy-2,2,6,6-tetramethylpiperidine-1-oxyl and iodobenzene derivatives in the presence of palladium(II) acetate coordinated with a tri(o-tolyl)phosphine ligand immobilized in a polyurea matrix.
\end{abstract}

\section{Introduction}

Cinnamic acid derivatives are known as biologically active compounds. Cinnamic acid and its hydroxy derivatives bearing a phenolic moiety such as coumaric, caffeic, ferulic, sinapinic, and chlorogenic acids [2-6], simple cinnamic acid derivatives (esters, amides, etc.), and prenylated cinnamates [4], have been proved to be effective as antioxidants [2,7], radical scavengers [2], antimicrobials [2,7,8], antibacterials [2], antivirals [2,7], and fungicides $[2,7,8]$. Cinnamic derivatives have also been recognized as active agents against tuberculosis and malaria $[3,7]$, cardiovascular diseases [3], and cancer [4]. Cinnamates show depigmenting [4], antidiabetic, antihyperglycemic, anticholesterolemic, anti-inflammatory, hepatoprotective, CNS depressant, anxiolytic, and cytotoxic activity [7]. Cinnamate esters have been used as effective UV filters (especially in UVB region, 280-320 nm) in cosmetics [7,9], and as fragrance materials [7]. Fragrance material reviews on cinnamic acid derivatives were extensively described in Food and Chemical Toxicology (especially in 2007) and in Food and Cosmetics Toxicology (in the 70s). The most recent reviews in this series were published in 2011 [10,11].

Cinnamic acid derivatives can be synthesized using Perkin, Knoevenagel, Claisen [7], and Wittig [12] condensation reactions. Since the discovery of catalytic coupling reactions, cinnamic derivatives have also been obtained using the Mizoroki-Heck cross-coupling reaction between aryl halides 
and an olefin [7,13-17]. Because cinnamates themselves are also olefins, they can serve as cinnamic substrates to synthesize more complex cinnamates $[18,19]$. Cinnamic acid derivatives are also formed when saturated aliphatic acids (instead of unsaturated ones as acrylates) are reacted with simple aromatic compounds (as benzene) in the presence of palladium(II) chloride [20]. Due to the important biological activity of cinnamates, the incorporation of a spin label moiety, particularly a nitroxyl fragment, is interesting, because it allows for the study of cinnamates using spin labeling methods.

To accomplish the synthesis of cinnamates bearing a nitroxyl moiety, we applied a Mizoroki-Heck cross-coupling reaction, using 4-acryloyloxy-2,2,6,6-tetramethylpiperidine-1-oxyl (3, an olefin component bearing a nitroxyl moiety), recently applied in the Morita-Baylis-Hillmann reaction [21]. The use of nitroxides in cross-coupling reactions is described only in a limited number of papers [22-32]. To the best of our knowledge, there are no systematic studies on the use of nitroxides in the Mizoroki-Heck cross-coupling reaction.

Despite the observation that an unpaired electron in nitroxides does not participate in organic reactions has been well known since the beginning of the nitroxide progress in 60's [33] the reactions of nitroxides involving an unpaired electron are also recognized (e.g., reductions, acidic medium, carbene addition, etc.). Because we would like to check the possibility of performing the Mizoroki-Heck cross-coupling reaction with nitroxides, herein the synthesis of cinnamates bearing a nitroxyl moiety is presented using aryl iodides as exemplary test compounds.

\section{Results and Disscusion}

4-Acryloyloxy-2,2,6,6-tetramethylpiperidine-1-oxyl (3) was obtained in $90-95 \%$ yield by the reaction of acryloyl chloride (2) with 2,2,6,6-tetramethyl-4-piperidinol-1-oxyl (1) in the presence of triethylamine [21,34-36].
The couplings of 4-acryloyloxy-2,2,6,6-tetramethylpiperidine1-oxyl (3) with iodobenzene (4a) and 4-methyliodobenzene (4b) were used as the test reactions to check the effectiveness of various palladium catalyst systems.

The use of $\mathrm{Pd}(\mathrm{OAc})_{2} / \mathrm{Ph}_{3} \mathrm{P} / \mathrm{Bu}_{3} \mathrm{~N}$ [37] resulted in a low yield of the target products. No products were obtained when other catalyst systems: $\mathrm{Pd}\left[\mathrm{PPh}_{3}\right]_{4}[38], \mathrm{Pd}\left(\mathrm{CF}_{3} \mathrm{COO}\right)_{2} / \operatorname{tri}(2$-furylphosphine) and $\mathrm{Pd}(\text { acetylacetonate })_{2} /$ tri(2-furylphosphine) [39] were tested. As a rule, unreacted 3 was identified, and always isolated. A number of unidentified products were detected by means of TLC.

Finally, the target cinnamates bearing a nitroxyl moiety (5a-i) were obtained using the coupling of 4-acryloyloxy-2,2,6,6-tetramethylpiperidine-1-oxyl (3) with a series of iodobenzene derivatives $(\mathbf{4 a}-\mathbf{i})$ in the presence of palladium(II) acetate coordinated with tri( $o$-tolyl)phosphine ligand immobilized in a polyurea matrix (commercially available as PdEnCatTOTP30 $\left.{ }^{\mathrm{TM}}\right)$ [40-45] (Scheme 1, Table 1).

The best results were obtained when an electron-withdrawing substituent $\mathrm{NO}_{2}$ was present in the benzene ring. The reactions of iodobenzene bearing 4-F, 4- $\mathrm{CF}_{3}, 2,4-\mathrm{diNO}_{2}$ substituents were unsuccessful. In the case of the reaction of $\mathbf{4 b}$ leading to the cinnamate $\mathbf{5 b}$, the results were unrepeatable (the times and yields of the reactions). Unidentified impurities together with the product $\mathbf{5 b}$ were observed $\left({ }^{13} \mathrm{C} \mathrm{NMR}\right)$.

No products in the case of 4-F, 4- $\mathrm{CF}_{3}, 2,4-\mathrm{diNO}_{2}$ substituents and unrepeatability in the case of cinnamate $\mathbf{5 b}$ can be caused by means of a type of a heterogeneous catalyst which is immobilized on a solid support. In addition to the widely highlighted unquestionable advantages of immobilized, heterogeneous catalysts (easy separation from the reaction mixture and the possibility of re-use), such catalysts are rigidly anchored on a carrier and may cause hindered interaction with substrates. Some of the<smiles>[R]C=CC(=O)/C=C/c1ccccc1-c1ccccc1I</smiles>

\begin{tabular}{l|l}
$\mathbf{4 , 5}$ & $\mathrm{R}$ \\
\hline $\mathbf{a}$ & $\mathrm{H}$ \\
$\mathbf{b}$ & $4-\mathrm{CH}_{3}$ \\
$\mathbf{c}$ & $3,4-\mathrm{diCl}$ \\
$\mathbf{d}$ & $4-\mathrm{CH}_{3} \mathrm{O}$ \\
e & $2-\mathrm{NO}_{2}$ \\
$\mathbf{f}$ & $3-\mathrm{NO}_{2}$ \\
$\mathbf{g}$ & $4-\mathrm{NO}_{2}$ \\
$\mathbf{h}$ & $2-\mathrm{CH}_{3}-4-\mathrm{NO}_{2}$ \\
$\mathbf{i}$ & $2-\mathrm{Cl}-4-\mathrm{NO}_{2}$
\end{tabular}




\begin{tabular}{|c|c|c|c|c|c|}
\hline Compound & $\mathrm{R}$ & Reaction temperature $\left[{ }^{\circ} \mathrm{C}\right]$ & Reaction time [h] & Yield [\%] & $\mathrm{mp}\left[{ }^{\circ} \mathrm{C}\right]$ \\
\hline $5 a$ & $\mathrm{H}$ & 80 & 22 & 42.4 & $97-98$ \\
\hline $5 b$ & $4-\mathrm{CH}_{3}$ & 100 & $2-20$ & 30.4 & oil \\
\hline $5 c$ & 3,4-diCl & 80 & 25 & 62.7 & $118-120$ \\
\hline $5 d$ & $4-\mathrm{CH}_{3} \mathrm{O}$ & 85 & 24 & 75.8 & $70-74$ \\
\hline $5 e$ & $2-\mathrm{NO}_{2}$ & 90 & 20 & 76.4 & $107-110$ \\
\hline $5 f$ & $3-\mathrm{NO}_{2}$ & 80-90 & 20 & 99.9 & $121-123$ \\
\hline $5 g$ & $4-\mathrm{NO}_{2}$ & $80-85$ & 23 & 55.3 & $148-149$ \\
\hline $5 h$ & $2-\mathrm{CH}_{3}-4-\mathrm{NO}_{2}$ & $80-85$ & 27 & 88.3 & $109-112$ \\
\hline $5 i$ & $2-\mathrm{Cl}-4-\mathrm{NO}_{2}$ & 80-85 & 27 & 56.2 & $107-110$ \\
\hline
\end{tabular}

catalytic sites may be buried within the polymer matrix and cannot participate in the reactions [46]. This disadvantage may cause various unwanted effects. As examples, no clear relationship between the structures of the aryl iodides and the yields were observed, as described in [47], or a catalyst that accepts only electron-rich aryl iodides [48].

The structure of the synthesized cinnamates bearing a nitroxyl moiety were confirmed using EIMS, ESIMS, HREIMS, HRESIMS, ${ }^{1} \mathrm{H}$ NMR, ${ }^{13} \mathrm{C}$ NMR, and IR spectra (Experimental part and Supporting Information File 1). Recording of the NMR spectra of the nitroxides required removing of the unpaired electron. This was achieved by adding a drop of a reducing agent - in fact the spectra of the corresponding hydroxylamines were recorded. In this research phenylhydrazine has been used $[49,50]$, however, sometimes using of hydrazobenzene [51,52] or pentafluorophenyl hydrazine [35] as reducing agents have been reported, as well.

The $E$ geometry of $\mathbf{5 a}-\mathbf{i}$ was confirmed by ${ }^{1} \mathrm{H}$ NMR spectroscopy. The values of the coupling constants of the doublets visible in the ${ }^{1} \mathrm{H}$ NMR spectra of $\mathbf{5 a}-\mathbf{i}$ belonging to the double bond of $5 \mathbf{a}-\mathbf{i}$ remain in the range $15.5-16.0 \mathrm{~Hz}$. These values confirm the $E$ geometry of $\mathbf{5 a}-\mathbf{i}$. In Table 2 the shifts and the coupling constants of the double bond are presented.

The substituent in 2-position of the cinnamates $5 \mathbf{e}, \mathbf{5 h}, \mathbf{5 i}$ causes the noticeable shift of $\mathrm{H}^{\mathrm{D}}$ hydrogen to the lower field. This observation suggests that conformation $\mathrm{X}$, where the substituent in 2-position and the $\mathrm{H}^{\mathrm{D}}$ atom are placed close to each other, is more populated than conformation Y (Figure 1).

For cinnamate $\mathbf{5} \mathbf{f}\left(3-\mathrm{NO}_{2}\right)$, the direct measurement of the chemical shift of the hydrogen atom $\mathrm{H}^{\mathrm{D}}\left(\mathrm{OOC}-\mathrm{CH}^{(\mathrm{B})}=\mathrm{CH}^{(\mathbf{D})}-\mathrm{C}_{6} \mathrm{H}_{4}-\right.$ $\mathrm{NO}_{2}{ }^{(\mathrm{m})}$ ) was not possible, due to the presence of more than one equidistant $(16 \mathrm{~Hz})$ peak close to $7.5 \mathrm{ppm}$. Irradiation of $\mathrm{H}^{\mathrm{B}}$ $(\delta 6.53 \mathrm{ppm})$ allowed to distinguish the signal of $\mathrm{H}^{\mathrm{D}}$ at $7.69 \mathrm{ppm}$.
Table 2: Chemical shifts and coupling constants of the hydrogen atoms belonging to the double bond of $5 \mathbf{a}-\mathbf{i}$ : $\mathrm{OOC}-\mathrm{CH}^{(\mathrm{B})}=\mathrm{CH}^{(\mathrm{D})}-\mathrm{C}_{6} \mathrm{H}_{4(3)}-\mathrm{R}$.

\begin{tabular}{|c|c|c|c|c|}
\hline 5 & $\mathrm{R}$ & $\delta \mathrm{H}^{(\mathrm{B})}[\mathrm{ppm}]$ & $\delta \mathrm{H}^{(\mathrm{D})}[\mathrm{ppm}]$ & $J_{\mathrm{DB}}[\mathrm{Hz}]$ \\
\hline $\mathbf{a}$ & $\mathrm{H}$ & 6.41 & 7.67 & 16.0 \\
\hline b & $4-\mathrm{CH}_{3}$ & 6.35 & 7.64 & 15.8 \\
\hline c & 3,4-diCl & 6.38 & 7.40 & 15.5 \\
\hline d & $\mathrm{OCH}_{3}$ & 6.28 & 7.62 & 16.0 \\
\hline e & $2-\mathrm{NO}_{2}$ & 6.33 & 8.10 & 16.0 \\
\hline f & $3-\mathrm{NO}_{2}$ & 6.53 & 7.69 & 16.0 \\
\hline g & $4-\mathrm{NO}_{2}$ & 6.52 & 7.32 & 16.0 \\
\hline $\mathbf{h}$ & $2-\mathrm{CH}_{3}-4-\mathrm{NO}_{2}$ & 6.42 & 7.90 & 15.9 \\
\hline i & $2-\mathrm{Cl}-4-\mathrm{NO}_{2}$ & 6.51 & 8.03 & 16.0 \\
\hline
\end{tabular}
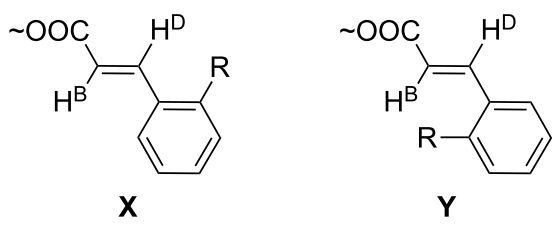

Figure 1: Conformations of the substituent in 2-position of the cinnamates $5 \mathbf{e}, \mathbf{5 h}, \mathbf{5 i}$.

A molecular peak was present in all mass spectra of the synthesized products $\mathbf{5 a}-\mathbf{5 i}$. Except in the case of $\mathbf{5 c}, \mathbf{i}$ it was clearly visible (5-25\%). In all of the mass spectra, the abundant peak of the cinnamyl acyl cation $\mathrm{ArCH}=\mathrm{CHCO}^{+}$was present. This was a base peak in the case of $\mathbf{5 a , d}$.

Characteristic signals for 4- $X O$-substituted 2,2,6,6-tetramethylpiperidine-1-oxyl moiety (TEMPOL, TEMPOL esters, etc.) were observed at $m / z=154,124,109$ [53,54]. The signals at $\mathrm{m} / \mathrm{z} 124$, and 109 are abundant. The signal at $\mathrm{m} / \mathrm{z} 154$ was assigned to the structure, resulting from elimination of a $\mathrm{XOH}$ from the position 4 and 3 of the piperidine ring. The subsequent loss of a $\mathrm{NO}$ group $(\mathrm{M}=30)$ and $\mathrm{CH}_{3}$ group $(\mathrm{M}=15)$, respectively, generates ions at $m / z 124$ and 109 (Scheme 2). The 
peaks at $\mathrm{m} / \mathrm{z} 154,124$ and 109 were thoroughly analyzed for the acrylate $\mathbf{3}$, as an example.

Proposed elemental composition of the fragmentation ions at $m / z 154,124,109$ for the acrylate 3 were confirmed by the measurement of their exact mass:

- $m / z 154$ (calculated $\mathrm{C}_{9} \mathrm{H}_{16} \mathrm{NO}$ : 154.12319, found: 154.12390)

- $m / z 124$ (calculated for $\mathrm{C}_{9} \mathrm{H}_{16}$ : 124.12520, found: 124.12515),

- $m / z 109$ (calculated for $\mathrm{C}_{8} \mathrm{H}_{13}: 109.10173$, found: 109.10079).

The signal at $m / z 124$ is the base one in the case of $\mathbf{5 b}, \mathbf{e}, \mathbf{g}, \mathbf{h}$, and that at $\mathrm{m} / \mathrm{z} 109$ in the case of $\mathbf{5} \mathbf{c}, \mathbf{f}, \mathbf{i}$. The $\mathrm{m} / \mathrm{z}$ values and relative intensities of the discussed signals are collected in Table 3.

In conclusion, we showed that 4-acryloyloxy-2,2,6,6-tetramethylpiperidine-1-oxyl (3) can be used as starting material in the synthesis of new cinnamates containing a nitroxyl group in a Mizoroki-Heck cross-coupling reaction.

\section{Experimental}

\section{General}

The protocols for the synthesis of 4-acryloyloxy-2,2,6,6-tetramethylpiperidine-1-oxyl (3) and its precursor 2,2,6,6-tetra- methyl-4-hydroxypiperidin-1-oxyl (TEMPOL, 1) were done according to [21]. The identity of $\mathbf{3}$ was additionally confirmed by ${ }^{1} \mathrm{H}$ and ${ }^{13} \mathrm{C}$ NMR performed in the presence of $\mathrm{PhNHNH}_{2}$. ${ }^{1} \mathrm{H}$ NMR $\left(200 \mathrm{MHz}, \delta, \mathrm{CDCl}_{3}, \mathrm{TMS}\right.$, in the presence of $\left.\mathrm{PhNHNH}_{2}\right) 1.23\left(\mathrm{~s}, 6 \mathrm{H},\left(\mathrm{CH}_{3}\right)\left(\mathrm{CH}_{3}\right) \mathrm{CN}(\mathrm{OH}) \mathrm{C}\left(\mathrm{CH}_{3}\right)\left(\mathrm{CH}_{3}\right)\right.$, $1.24\left(\mathrm{~s}, 6 \mathrm{H},\left(\mathrm{CH}_{3}\right)\left(\mathrm{CH}_{3}\right) \mathrm{CN}(\mathrm{OH}) \mathrm{C}\left(\mathrm{CH}_{3}\right)\left(\mathrm{CH}_{3}\right)\right), 1.65(\mathrm{t}, J=$ $11.8 \mathrm{~Hz}, 2 \mathrm{H}, H \mathrm{HC}-\mathrm{CH}(\mathrm{O}-)-\mathrm{CH} H), 1.96$ (ddt, $J=12.8 \mathrm{~Hz}, J=$ $4.2 \mathrm{~Hz}, J=1.5 \mathrm{~Hz}, 2 \mathrm{H}, \mathrm{H} H \mathrm{C}-\mathrm{CH}(\mathrm{O}-)-\mathrm{C} H \mathrm{H}), 5.13$ (tt, $J=11.2$ $\mathrm{Hz}, J=4.3 \mathrm{~Hz}, 1 \mathrm{H}, \mathrm{C} H-\mathrm{OC}(=\mathrm{O})), 5.81(\mathrm{dd}, J=10.3 \mathrm{~Hz}, J=1.6$ $\mathrm{Hz}, 1 \mathrm{H}, \mathrm{CHH}=\mathrm{CH}-\mathrm{COO}($ cis $)), 6.09(\mathrm{dd}, J=17.2 \mathrm{~Hz}, J=10.3$ $\mathrm{Hz}, 1 \mathrm{H}, \mathrm{CH}_{2}=\mathrm{CH}$-COO), 6.39 (dd, $J=17.2 \mathrm{~Hz}, J=1.6 \mathrm{~Hz}, 1 \mathrm{H}$, $\mathrm{CH} H=\mathrm{CH}-\mathrm{COO}($ trans $)) ;{ }^{13} \mathrm{C} \mathrm{NMR}\left(50 \mathrm{MHz}, \delta, \mathrm{CDCl}_{3}, \mathrm{TMS}\right.$, in the presence of $\left.\mathrm{PhNHNH}_{2}\right) 20.79\left(2 \times \mathrm{CH}_{3}\right), 31.86\left(2 \times \mathrm{CH}_{3}\right)$, $43.92\left(2 \times \mathrm{CH}_{2}\right), 59.89\left(2 \times \mathrm{C}\left(\mathrm{CH}_{3}\right)_{2}\right), 66.99(\mathrm{CH}-\mathrm{O}-), 128.94$ $\left(\mathrm{COCH}=\mathrm{CH}_{2}\right), 130.84\left(\mathrm{COCH}=\mathrm{CH}_{2}\right), 165.95(\mathrm{C}=\mathrm{O})$.

PdEnCatTOTP30 was purchased from Aldrich. Iodobenzene derivatives and catalysts were purchased from Aldrich, AlfaAesar and Fluorochem. The $\mathrm{Pd}\left(\mathrm{PPh}_{3}\right)_{4}$ was synthesized according to the known procedure [38]. The experiments were performed in a $25-50 \mathrm{~mL}$ round-bottom two necked flask, equipped with a magnetic stirrer and a reflux condenser under anhydrous argon atmosphere (inlet through the top of the condenser equipped with a short drying column packed with drying silica, outlet by a needle placed in a septum in a side neck of the flask). Most of the products were obtained as red solids. TLC was performed on silica gel Merck aluminium roll<smiles>C=CC(=O)OC1CC(C)(C)N(C(C)(C)C)C(C)(C)C1C(=O)O</smiles>

Scheme 2: The formation of the fragment ions at $\mathrm{m} / \mathrm{z} 154,124,109$.

Table 3: The relative intensities of the molecular, cinnamic acyl, $m / z$ 154, $m / z$ 124, and $m / z 109$ ions.

(a)

$\begin{array}{lllll} & 302(10) & 131\left(100, \mathrm{C}_{6} \mathrm{H}_{5} \mathrm{CH}=\mathrm{CHCO}\right) & 27 & 89\end{array}$

b $\quad 316(24)$

c $\quad 370(2)$

d $332(19)$

e $\quad 347(23)$

f $\quad 347(5)$

g $347(19)$

h $\quad 361(15)$

i $381(2)$

\section{$145\left(91,4-\mathrm{CH}_{3} \mathrm{C}_{6} \mathrm{H}_{4} \mathrm{CH}=\mathrm{CHCO}\right)$}

$199\left(64,3,4-\mathrm{Cl}_{2} \mathrm{CH}_{3} \mathrm{C}_{6} \mathrm{H}_{3} \mathrm{CH}=\mathrm{CHCO}\right)$

$161\left(100,4-\mathrm{CH}_{3} \mathrm{OC}_{6} \mathrm{H}_{4} \mathrm{CH}=\mathrm{CHCO}\right)$

$176\left(63,2-\mathrm{NO}_{2} \mathrm{C}_{6} \mathrm{H}_{4} \mathrm{CH}=\mathrm{CHCO}\right)$

$176\left(85,3-\mathrm{NO}_{2} \mathrm{C}_{6} \mathrm{H}_{4} \mathrm{CH}=\mathrm{CHCO}\right)$

$176\left(89,4-\mathrm{NO}_{2} \mathrm{C}_{6} \mathrm{H}_{4} \mathrm{CH}=\mathrm{CHCO}\right)$

$190\left(74,2-\mathrm{CH}_{3}-4-\mathrm{NO}_{2} \mathrm{C}_{6} \mathrm{H}_{4} \mathrm{CH}=\mathrm{CHCO}\right)$

$210\left(41,2-\mathrm{Cl}-4-\mathrm{NO}_{2} \mathrm{C}_{6} \mathrm{H}_{4} \mathrm{CH}=\mathrm{CHCO}\right)$

$\begin{array}{lll}46 & 100 & 75 \\ 16 & 88 & 100 \\ 20 & 61 & 44 \\ 25 & 100 & 87 \\ 13 & 83 & 100 \\ 19 & 100 & 90 \\ 29 & 100 & 82 \\ 15 & 89 & 100\end{array}$


5562 or aluminium sheets 5554 . Column chromatography was performed using Merck 1.09385.1000 or Zeochem 60 hyd $40-63 \mu \mathrm{m}$ silica gel $(0.040-0.063 \mathrm{~mm}, 230-400 \mathrm{mesh})$, respectively. TLC visualisation: UV $254 \mathrm{~nm}$ light and/or iodine vapours. MS (EI, $70 \mathrm{eV}, \mathrm{m} / \mathrm{z}$, int. (\%)) data were recorded using AMD 604 and Agilent Technologies 5975 B mass spectrometers. HRMS (EI) data were recorded using an AMD 604 mass spectrometer. $\mathrm{MS}$ and HRMS (ESI, positive ion, $\mathrm{CH}_{3} \mathrm{OH}$ as a solvent) were recorded using a Micromass LCT apparatus. IR $\left(\mathrm{cm}^{-1}\right)$ data were recorded using a FTIR Jasco 420 spectrometer. ${ }^{1} \mathrm{H}$ and ${ }^{13} \mathrm{C}$ NMR data were collected using a Varian UNITYplus 200 spectrometer. NMR spectra were performed with a drop of phenylhydrazine (in fact the spectra of corresponding hydroxylamines were recorded) [49].

\section{Cinnamates $\mathbf{5 a - i}$; general procedure using PdEnCatTOTP30 as a catalyst}

4-Acryloyloxy-2,2,6,6-tetramethylpiperidin-1-oxyl (3, $0.113 \mathrm{~g}$, $0.5 \mathrm{mmol}$ ), Aryl iodide $(\mathbf{4 a}-\mathbf{i}, 0.5 \mathrm{mmol}), \mathrm{Bu}_{4} \mathrm{~N}^{+} \mathrm{AcO}^{-}$(hygroscopic) (0.3 g), PdEnCatTOTP30 (0.0625 g, $0.025 \mathrm{~mol}$, $5 \mathrm{~mol} \%$ ), and toluene $(2 \mathrm{~mL})$ were placed in a flask, stirred and heated at $80-100{ }^{\circ} \mathrm{C}$ for $20-27 \mathrm{~h}$ under argon. The particular temperatures and times of the reactions are summarized in Table 1. After approximately $6 \mathrm{~h}$, a second portion of a catalyst $(0.0625 \mathrm{~g}, 0.025 \mathrm{~mol}, 5 \mathrm{~mol} \%)$ was added. The progress of the reaction was monitored by TLC (silica, hexane/ethyl acetate 9:1). Upon completion of the reaction, the solids were filtered off, the dark filtrate was concentrated under reduced pressure and subjected to column chromatography (hexane/ethyl acetate 9:1, benzene/ethyl acetate 95:5, benzene/methanol 95:5 as possible mobile phases). The red zone was collected, the eluate was evaporated under reduced pressure to yield red oils (solidified in a refrigerator), or directly red crystals of $\mathbf{5 a}-\mathbf{i}$.

\section{2,2,6,6-Tetramethyl-1-oxyl-4-piperidyl 3-E- phenylacrylate (5a)}

42.4\%; mp 97-98 ${ }^{\circ} \mathrm{C}$; MS (EI, $70 \mathrm{eV}, \mathrm{m} / \mathrm{z}$, int [\%]) 302 (10, $\mathrm{M}^{+}$), 272 (7), 207 (19), 179 (6), 178 (10), 154 (27), 140 (20), 139 (14), 131 (100, $\mathrm{ArCH}=\mathrm{CHCO}), 124$ (89), 109 (69), 103 (41), 82 (15), 81 (12), 77 (21), 69 (13), 68 (9), 67 (9), 55 (10), 41 (18); HRMS (EI, $70 \mathrm{eV}, m / z$, int [\%]): calcd for $\mathrm{C}_{18} \mathrm{H}_{24} \mathrm{NO}_{3}$ : 302.1756, found: 302.1750; ${ }^{1} \mathrm{H} \mathrm{NMR}\left(200 \mathrm{MHz}, \delta, \mathrm{CDCl}_{3}\right.$, TMS, $\left.\mathrm{PhNHNH}_{2}\right) 1.26\left(\mathrm{~s}, 6 \mathrm{H},\left(\mathrm{CH}_{3}\right)\left(\mathrm{CH}_{3}\right) \mathrm{CN}(\mathrm{OH})-\right.$ $\mathrm{C}\left(\mathrm{CH}_{3}\right)\left(\mathrm{CH}_{3}\right), 1.28\left(\mathrm{~s}, 6 \mathrm{H},\left(\mathrm{CH}_{3}\right)\left(\mathrm{CH}_{3}\right) \mathrm{CN}(\mathrm{OH}) \mathrm{C}\left(\mathrm{CH}_{3}\right)\left(\mathrm{CH}_{3}\right)\right)$, $1.74(\mathrm{t}, J=12.0 \mathrm{~Hz}, 2 \mathrm{H}, H \mathrm{HC}-\mathrm{CH}(\mathrm{O}-)-\mathrm{CH} H), 2.02$ (ddd, $J=$ $12.8 \mathrm{~Hz}, J=4.4 \mathrm{~Hz}, J=1.2 \mathrm{~Hz}, 2 \mathrm{H}, \mathrm{H} H \mathrm{C}-\mathrm{CH}(\mathrm{O}-)-\mathrm{C} H \mathrm{H}), 5.20$ (tt, $J=11.2 \mathrm{~Hz}, J=4.3 \mathrm{~Hz}, 1 \mathrm{H}, \mathrm{CH}-\mathrm{OC}(=\mathrm{O})), 6.41(\mathrm{~d}, J=16.0$ $\mathrm{Hz}, 1 \mathrm{H}, \mathrm{Ar}-\mathrm{CH}=\mathrm{CH}-\mathrm{COO}), 7.67(\mathrm{~d}, J=16.0 \mathrm{~Hz}, 1 \mathrm{H}$, $\mathrm{Ar}-\mathrm{C} H=\mathrm{CH}-\mathrm{COO}) ;{ }^{13} \mathrm{C} \mathrm{NMR}\left(50 \mathrm{MHz}, \delta, \mathrm{CDCl}_{3}, \mathrm{TMS}\right.$, $\left.\mathrm{PhNHNH}_{2}\right) 20.87\left(2 \times \mathrm{CH}_{3}\right), 31.67\left(2 \times \mathrm{CH}_{3}\right), 43.87\left(2 \times \mathrm{CH}_{2}\right)$, $60.46\left(2 \times C\left(\mathrm{CH}_{3}\right)_{2}\right), 66.76(\mathrm{CH}-\mathrm{O}-), 118.51(\mathrm{CH}), 128.30(\mathrm{CH})$,
$129.10(\mathrm{CH}), 130.52(\mathrm{CH}), 134.57(\mathrm{C}), 145.00(\mathrm{CH}), 166.68$ $(\mathrm{C}=\mathrm{O})$; IR $\left(\mathrm{cm}^{-1}, \mathrm{KBr}\right) 2976,2937,1712,1639,1450,1308$, 1168, 1008, 978, 859, 765, 709, 685.

\section{2,2,6,6-Tetramethyl-1-oxyl-4-piperidyl 3-E-(4- methylphenyl)acrylate (5b)}

$30.4 \%$, oil; MS (EI, $70 \mathrm{eV}, \mathrm{m} / z$, int [\%]) $316\left(24, \mathrm{M}^{+}\right), 286(15)$, 230 (6), 163 (13), 162 (21), 154 (46), 145 (91, $\mathrm{ArCH}=\mathrm{CHCO})$, 140 (62), 139 (27), 124 (100), 117 (43), 115 (49), 109 (75), 98 (14), 91 (32), 82 (37), 81 (22), 69 (31), 68 (17), 67 (18), 65 (10), 60 (8), 59 (9), 58 (11), 57 (13), 56 (19), 55 (25), 43 (35), 41 (39); MS (ESI, $m / z$, int [\%]): $340(15, \mathrm{M}+23+\mathrm{H}), 318(\mathrm{M}$ $+2 \mathrm{H}$ ); HRMS (EI, $70 \mathrm{eV}, m / z$, int [\%]): calcd for $\mathrm{C}_{19} \mathrm{H}_{26} \mathrm{NO}_{3}$ : 316.1913, found: 316.1926; ${ }^{1} \mathrm{H} \mathrm{NMR}\left(200 \mathrm{MHz}, \delta, \mathrm{CDCl}_{3}\right.$, TMS, $\left.\mathrm{PhNHNH}_{2}\right) 1.85$ (t, $J=12.1 \mathrm{~Hz}, 2 \mathrm{H}, H \mathrm{HC}-\mathrm{CH}(\mathrm{O}-)-$ $\mathrm{CH} H$ ), 2.02-2.17 (m, 2H, HHC-CH(O-)-CHH), 2.37 (s, 3H, $\left.\mathrm{CH}_{3}\right), 5.23(\mathrm{tt}, J=11.1 \mathrm{~Hz}, J=4.4 \mathrm{~Hz}, 1 \mathrm{H}, \mathrm{CH}-\mathrm{OC}(=\mathrm{O})), 6.35$ (d, $J=15.8 \mathrm{~Hz}, 1 \mathrm{H}, \mathrm{Ar}-\mathrm{CH}=\mathrm{CH}-\mathrm{COO}), 7.64(\mathrm{~d}, J=15.8 \mathrm{~Hz}$, $1 \mathrm{H}, \mathrm{Ar}-\mathrm{CH}=\mathrm{CH}-\mathrm{COO}) ;{ }^{13} \mathrm{C} \mathrm{NMR}\left(50 \mathrm{MHz}, \delta, \mathrm{CDCl}_{3}, \mathrm{TMS}\right.$, $\left.\mathrm{PhNHNH}_{2}\right): 21.35$ (impurity), $21.69\left(2 \times \mathrm{CH}_{3}\right), 23.04\left(\mathrm{ArCH}_{3}\right)$, $29.84\left(2 \times \mathrm{CH}_{3}\right), 43.01\left(2 \times \mathrm{CH}_{2}\right), 62.60\left(2 \times \mathrm{C}_{\left.\left(\mathrm{CH}_{3}\right)_{2}\right), 65.67}\right.$ (CH-O-), 115.32 (impurity), $117.03(\mathrm{CH}), 128.34(\mathrm{CH}), 129.86$ $(\mathrm{CH}), 141.12(\mathrm{C}), 145.37(\mathrm{CH}), 166.73(\mathrm{C}=\mathrm{O}), 179.47$ (impurity); IR (cm ${ }^{-1}$, film): 2976, 1711, 1635, 1166, 815.

\section{2,2,6,6-Tetramethyl-1-oxyl-4-piperidyl 3-E- (3,4-dichlorophenyl)acrylate (5c)}

62.7\%; pink crystals, mp $118-120^{\circ} \mathrm{C}$; MS (EI, $70 \mathrm{eV}, \mathrm{m} / \mathrm{z}$, int [\%]) $370\left(2, \mathrm{M}^{+}\right), 201$ (40), 199 (64, $\left.\mathrm{ArCH}=\mathrm{CHCO}\right), 173$ (12), 171 (19), 154 (16), 136 (42), 124 (88), 109 (100), 101 (8), 99 (11), 82 (16), 81 (19), 69 (18), 67 (21), 56 (17), 55 (25), 41 (65); MS (ESI, $m / z$, int [\%]): 395 (20), 393 (90, M + Na), 304 (100); HRMS (ESI): calcd for $\mathrm{C}_{18} \mathrm{H}_{22} \mathrm{NO}_{3} \mathrm{Cl}_{2} \mathrm{Na}$ : 393.0874 , found, 393.0890; ${ }^{1} \mathrm{H} \mathrm{NMR}\left(200 \mathrm{MHz}, \delta, \mathrm{CDCl}_{3}\right.$, TMS, $\left.\mathrm{PhNHNH}_{2}\right) 1.27\left(\mathrm{~s}, 6 \mathrm{H},\left(\mathrm{CH}_{3}\right)\left(\mathrm{CH}_{3}\right) \mathrm{CN}(\mathrm{OH}) \mathrm{C}\left(\mathrm{CH}_{3}\right)\left(\mathrm{CH}_{3}\right)\right)$, $1.29\left(\mathrm{~s}, 6 \mathrm{H},\left(\mathrm{CH}_{3}\right)\left(\mathrm{CH}_{3}\right) \mathrm{CN}(\mathrm{OH}) \mathrm{C}\left(\mathrm{CH}_{3}\right)\left(\mathrm{CH}_{3}\right)\right), 1.75(\mathrm{t}, J=$ $12.0 \mathrm{~Hz}, 2 \mathrm{H}, H \mathrm{HC}-\mathrm{CH}(\mathrm{O}-)-\mathrm{CH} H), 2.02(\mathrm{dd}, J=12.8 \mathrm{~Hz}, J=$ $4.4 \mathrm{~Hz}, 2 \mathrm{H}, \mathrm{H} H \mathrm{C}-\mathrm{CH}(\mathrm{O}-)-\mathrm{CHH}), 5.20$ (tt, $J=11.3 \mathrm{~Hz}, J=4.3$ $\mathrm{Hz}, 1 \mathrm{H}, \mathrm{CH}-\mathrm{OC}(=\mathrm{O})), 6.38(\mathrm{~d}, J=15.5 \mathrm{~Hz}, 1 \mathrm{H}, \mathrm{Ar}-\mathrm{CH}=\mathrm{CH}-$ COO), 7.40 (d, $J=15.5 \mathrm{~Hz}, 1 \mathrm{H}, \mathrm{Ar}-\mathrm{CH}=\mathrm{CH}-\mathrm{COO}) ;{ }^{13} \mathrm{C} \mathrm{NMR}$ $\left(50 \mathrm{MHz}, \delta, \mathrm{CDCl}_{3}, \mathrm{TMS}, \mathrm{PhNHNH}_{2}\right) 20.87\left(2 \times \mathrm{CH}_{3}\right), 31.54$ $\left(2 \times \mathrm{CH}_{3}\right), 43.72\left(2 \times \mathrm{CH}_{2}\right), 60.70\left(2 \times C\left(\mathrm{CH}_{3}\right)_{2}\right), 67.04(\mathrm{CH}-\mathrm{O}-)$, $117.10(\mathrm{C}), 120.36(\mathrm{CH}), 127.23(\mathrm{CH}), 129.85(\mathrm{CH}), 131.10$ $(\mathrm{CH}), 133.45(\mathrm{C}), 134.59(\mathrm{C}), 142.24(\mathrm{CH}), 166.02(\mathrm{C}=\mathrm{O})$; IR $\left(\mathrm{cm}^{-1}, \mathrm{KBr}\right) 2975,1706,1638,1474,1319,1267,1173,1150$, $1206,979,822$.

\section{2,2,6,6-Tetramethyl-1-oxyl-4-piperidyl 3-E-(4- methoxyphenyl)acrylate (5d)}

$75.8 \%$; mp $70-74{ }^{\circ} \mathrm{C}$; MS (EI, $70 \mathrm{eV}, \mathrm{m} / z$, int [\%]) 332 (19, $\mathrm{M}^{+}$), 302 (6), 284 (2), 267 (5), 246 (4), 178 (32), 161 (100, 
$\mathrm{ArCH}=\mathrm{CHCO}), 154$ (20), 140 (8), 139 (9), 133 (17), 124 (61), 109 (44); MS (ESI, $m / z$, int [\%]): 355 (100, M + Na); HRMS (ESI): calcd for $\mathrm{C}_{19} \mathrm{H}_{26} \mathrm{NO}_{4} \mathrm{Na}$ : 355.1760, found, 355.1753; ${ }^{1} \mathrm{H}$ NMR (200 MHz, $\left.\delta, \mathrm{CDCl}_{3}, \mathrm{TMS}, \mathrm{PhNHNH}_{2}\right) 1.24(\mathrm{~s}, 6 \mathrm{H}$, $\left.\left(\mathrm{CH}_{3}\right)\left(\mathrm{CH}_{3}\right) \mathrm{CN}(\mathrm{OH}) \mathrm{C}\left(\mathrm{CH}_{3}\right)\left(\mathrm{CH}_{3}\right)\right), 1.25(\mathrm{~s}, 6 \mathrm{H}$, $\left.\left(\mathrm{CH}_{3}\right)\left(\mathrm{CH}_{3}\right) \mathrm{CN}(\mathrm{OH}) \mathrm{C}\left(\mathrm{CH}_{3}\right)\left(\mathrm{CH}_{3}\right)\right), 1.67(\mathrm{t}, J=11.9 \mathrm{~Hz}, 2 \mathrm{H}$, $H \mathrm{HC}-\mathrm{CH}(\mathrm{O}-)-\mathrm{CH} H$ ), 2.00 (ddd, $J=11.2 \mathrm{~Hz}, J=4.4 \mathrm{~Hz}, J=1.4$ $\mathrm{Hz}, 2 \mathrm{H}, \mathrm{H} H \mathrm{C}-\mathrm{CH}(\mathrm{O}-)-\mathrm{CHH}), 1.67$ (t, $J=11.9 \mathrm{~Hz}, 2 \mathrm{H}, H \mathrm{HC}-$ CHOCO-CHH), 2.00 (ddd, $J=1.4 \mathrm{~Hz}, J=4.4 \mathrm{~Hz}, J=11.2 \mathrm{~Hz}$, $2 \mathrm{H}, \mathrm{H} H \mathrm{C}-\mathrm{CHOCO}-\mathrm{CHH}), 3.83\left(\mathrm{~s}, 3 \mathrm{H}, \mathrm{OCH}_{3}\right), 5.18(\mathrm{tt}, J=$ $11.2 \mathrm{~Hz}, J=4.4 \mathrm{~Hz}, 1 \mathrm{H}, \mathrm{CH}-\mathrm{OC}(=\mathrm{O})), 6.28(\mathrm{~d}, J=16.0 \mathrm{~Hz}$, $1 \mathrm{H}, \mathrm{Ar}-\mathrm{CH}=\mathrm{CH}-\mathrm{COO}), 7.62(\mathrm{~d}, J=16.0 \mathrm{~Hz}, 1 \mathrm{H}, \mathrm{Ar}-\mathrm{CH}=\mathrm{CH}-$ $\mathrm{COO}) ;{ }^{13} \mathrm{C} \mathrm{NMR}\left(50 \mathrm{MHz}, \delta, \mathrm{CDCl}_{3}, \mathrm{TMS}, \mathrm{PhNHNH}_{2}\right) 20.79$ $\left(2 \times \mathrm{CH}_{3}\right), 31.96\left(2 \times \mathrm{CH}_{3}\right), 44.12\left(2 \times \mathrm{CH}_{2}\right), 55.57\left(\mathrm{OCH}_{3}\right), 59.90$ $\left(2 \times C\left(\mathrm{CH}_{3}\right)_{2}\right), 66.68(\mathrm{CH}-\mathrm{O}-), 114.51(\mathrm{CH}), 116.03(\mathrm{CH})$, 127.33, $129.94(\mathrm{CH}), 144.60(\mathrm{CH}), 161.57,167.02(\mathrm{C}=\mathrm{O})$; IR $\left(\mathrm{cm}^{-1}, \mathrm{KBr}\right) 1707,1632,1604,1515,1290,1255,1163,984$, 827.

\section{2,2,6,6-Tetramethyl-1-oxyl-4-piperidyl 3-E-(2- nitrophenyl)acrylate (5e)}

76.4\%; mp 107-110 ${ }^{\circ} \mathrm{C}$; MS (EI, $70 \mathrm{eV}, \mathrm{m} / z$, int [\%]) 347 (23, $\left.\mathrm{M}^{+}\right), 317$ (5), 176 (63, $\left.\mathrm{ArCH}=\mathrm{CHCO}\right), 154$ (25), 140 (11), 139 (17), 130 (61), 124 (100), 109 (87), 102 (21); MS (ESI, $m / z$, int [\%]): $370(100, \mathrm{M}+\mathrm{Na})$; HRMS (ESI): calcd for $\mathrm{C}_{18} \mathrm{H}_{23} \mathrm{~N}_{2} \mathrm{O}_{5} \mathrm{Na}: 370.1505$, found, 370.1492; ${ }^{1} \mathrm{H}$ NMR (200 $\left.\mathrm{MHz}, \delta, \mathrm{CDCl}_{3}, \mathrm{TMS}, \mathrm{PhNHNH}_{2}\right) 1.24(\mathrm{~s}, 6 \mathrm{H}$, $\left.\left(\mathrm{CH}_{3}\right)\left(\mathrm{CH}_{3}\right) \mathrm{CN}(\mathrm{OH}) \mathrm{C}\left(\mathrm{CH}_{3}\right)\left(\mathrm{CH}_{3}\right)\right), 1.25(\mathrm{~s}, 6 \mathrm{H}$, $\left.\left(\mathrm{CH}_{3}\right)\left(\mathrm{CH}_{3}\right) \mathrm{CN}(\mathrm{OH}) \mathrm{C}\left(\mathrm{CH}_{3}\right)\left(\mathrm{CH}_{3}\right)\right), 1.69(\mathrm{t}, J=11.8 \mathrm{~Hz}, 2 \mathrm{H}$, $H \mathrm{HC}-\mathrm{CH}(\mathrm{O}-)-\mathrm{CH} H), 2.01(\mathrm{ddd}, J=12.0 \mathrm{~Hz}, J=3.4 \mathrm{~Hz}, J=1.3$ $\mathrm{Hz}, 2 \mathrm{H}, \mathrm{H} H \mathrm{C}-\mathrm{CH}(\mathrm{O}-)-\mathrm{CHH}), 1.69$ (t, $J=11.8 \mathrm{~Hz}, 2 \mathrm{H}, H \mathrm{HC}-$ CHOCO-CHH), $2.01(\mathrm{dtd}, J=1.4 \mathrm{~Hz}, J=3.4 \mathrm{~Hz}, J=12.0 \mathrm{~Hz}$, $2 \mathrm{H}, \mathrm{H} H \mathrm{C}-\mathrm{CHOCO}-\mathrm{CHH}), 5.20$ (tt, $J=11.3 \mathrm{~Hz}, J=4.3 \mathrm{~Hz}, 1 \mathrm{H}$, $\mathrm{CH}-\mathrm{OC}(=\mathrm{O})), 6.33(\mathrm{~d}, J=16.0 \mathrm{~Hz}, 1 \mathrm{H}, \mathrm{Ar}-\mathrm{CH}=\mathrm{C} H-\mathrm{COO})$, $8.10(\mathrm{~d}, J=16.0 \mathrm{~Hz}, 1 \mathrm{H}, \mathrm{Ar}-\mathrm{C} H=\mathrm{CH}-\mathrm{COO}) ;{ }^{13} \mathrm{C}$ NMR $(50$ $\left.\mathrm{MHz}, \delta, \mathrm{CDCl}_{3}, \mathrm{TMS}, \mathrm{PhNHNH}_{2}\right) 20.82\left(2 \times \mathrm{CH}_{3}\right) 31.91$ $\left(2 \times \mathrm{CH}_{3}\right) 43.96\left(2 \times \mathrm{CH}_{2}\right) 59.84\left(2 \times \mathrm{C}_{\left.\left(\mathrm{CH}_{3}\right)_{2}\right)} 67.55(\mathrm{CH}-\mathrm{O})\right.$ $123.60(\mathrm{CH}) 125.14(\mathrm{CH}) 129.34(\mathrm{C}) 130.49(\mathrm{CH}) 130.80(\mathrm{C})$ $133.73(\mathrm{CH}) 140.27(\mathrm{CH}) 148.00(\mathrm{CH}) 165.51(\mathrm{C}=\mathrm{O})$; IR $\left(\mathrm{cm}^{-1}, \mathrm{KBr}\right) 2977,1717,1632,1523,1341,1292,1272,1171$, 1009, 980, 866, 789, 758 .

\section{2,2,6,6-Tetramethyl-1-oxyl-4-piperidyl 3-E-(3- nitrophenyl)acrylate (5f)}

99.9\%; mp 121-123 ${ }^{\circ} \mathrm{C}$; MS (EI, $70 \mathrm{eV}, \mathrm{m} / \mathrm{z}$, int [\%]) 347 (5, $\left.\mathrm{M}^{+}\right), 176$ (85, $\left.\mathrm{ArCH}=\mathrm{CHCO}\right), 160$ (6), 154 (13), 130 (17), 129 (17), 124 (83), 118 (12), 109 (100), 102 (73), 91 (13), 82 (17), 81 (22), 76 (21), 69 (18), 68 (12), 67 (25), 57 (14), 56 (18), 55 (27), 43 (19). 41 (62); MS (ESI, $m / z$, int [\%]): $370(100, \mathrm{M}+$ $\mathrm{Na}$ ); HRMS (ESI): calcd for $\mathrm{C}_{18} \mathrm{H}_{23} \mathrm{~N}_{2} \mathrm{O}_{5} \mathrm{Na}: 370.1505$, found, 370.1489; ${ }^{1} \mathrm{H}$ NMR (200 MHz, $\delta, \mathrm{CDCl}_{3}, \mathrm{TMS}, \mathrm{PhNHNH}_{2}$ ), $1.249\left(\mathrm{~s}, 6 \mathrm{H},\left(\mathrm{CH}_{3}\right)\left(\mathrm{CH}_{3}\right) \mathrm{CN}(\mathrm{OH}) \mathrm{C}\left(\mathrm{CH}_{3}\right)\left(\mathrm{CH}_{3}\right)\right), 1.254(\mathrm{~s}, 6 \mathrm{H}$, $\left.\left(\mathrm{CH}_{3}\right)\left(\mathrm{CH}_{3}\right) \mathrm{CN}(\mathrm{OH}) \mathrm{C}\left(\mathrm{CH}_{3}\right)\left(\mathrm{CH}_{3}\right)\right), 1.69(\mathrm{t}, J=12.0 \mathrm{~Hz}, 2 \mathrm{H}$, $H \mathrm{HC}-\mathrm{CHOCO}-\mathrm{CH} H), 2.01(\mathrm{ddd}, J=1.4 \mathrm{~Hz}, J=4.4 \mathrm{~Hz}, J=$ $11.2 \mathrm{~Hz}, 2 \mathrm{H}, \mathrm{H} H \mathrm{C}-\mathrm{CHOCO}-\mathrm{C} H \mathrm{H}), 5.21(\mathrm{tt}, J=11.4 \mathrm{~Hz}, J=$ $4.4 \mathrm{~Hz}, 1 \mathrm{H}, \mathrm{CH}-\mathrm{OC}(=\mathrm{O})), 6.53(\mathrm{~d}, J=16.0 \mathrm{~Hz}, 1 \mathrm{H}$, $\mathrm{Ar}-\mathrm{CH}=\mathrm{CH}-\mathrm{COO}), 7.69(\mathrm{~d}, J=16.0 \mathrm{~Hz}, 1 \mathrm{H}, \mathrm{Ar}-\mathrm{CH}=\mathrm{CH}-$ $\mathrm{COO}) ;{ }^{13} \mathrm{C}$ NMR (50 MHz, $\left.\delta, \mathrm{CDCl}_{3}, \mathrm{TMS}, \mathrm{PhNHNH}_{2}\right) 20.75$ $\left(2 \times \mathrm{CH}_{3}\right), 32.05\left(2 \times \mathrm{CH}_{3}\right), 44.05\left(2 \times \mathrm{CH}_{2}\right), 59.76\left(2 \times \mathrm{C}\left(\mathrm{CH}_{3}\right)_{2}\right)$, 67.55 (CH-O-), $121.77(\mathrm{CH}), 122.63(\mathrm{CH}), 124.74(\mathrm{CH})$, $130.16(\mathrm{CH}), 133.85(\mathrm{CH}), 136.32(\mathrm{C}), 142.00(\mathrm{CH}), 148.87$ (C), $165.83(\mathrm{C}=\mathrm{O})$; IR $\left(\mathrm{cm}^{-1}, \mathrm{KBr}\right) 3080,2971,1709,1642$, 1526, 1358, 1330, 1191, 983, 813, 671 .

\section{2,2,6,6-Tetramethyl-1-oxyl-4-piperidyl 3-E-(4- nitrophenyl)acrylate $\mathbf{( 5 g})$}

55.3\%; mp 148-149 ${ }^{\circ} \mathrm{C}$; MS (EI, $70 \mathrm{eV}, \mathrm{m} / z$, int [\%]) 347 (19, $\left.\mathrm{M}^{+}\right), 317$ (6), 261 (3), 176 (89, $\left.\mathrm{ArCH}=\mathrm{CHCO}\right), 160$ (7), 154 (19), 140 (14), 139 (15), 130 (24), 124 (100), 109 (90), 102 (29); HRMS (EI, $70 \mathrm{eV}, \mathrm{m} / z$, int [\%]): calcd for $\mathrm{C}_{18} \mathrm{H}_{23} \mathrm{~N}_{2} \mathrm{O}_{5}$ : 347.16070, found, 347.16009; ${ }^{1} \mathrm{H} \mathrm{NMR}\left(200 \mathrm{MHz}, \delta, \mathrm{CDCl}_{3}\right.$, TMS, $\left.\mathrm{PhNHNH}_{2}\right) 1.25\left(\mathrm{~s}, 12 \mathrm{H}, 4 \times \mathrm{CH}_{3}\right), 1.68(\mathrm{t}, J=12.0 \mathrm{~Hz}$, $2 \mathrm{H}, H \mathrm{HC}-\mathrm{CH}(\mathrm{O}-)-\mathrm{CH} H), 2.00(\mathrm{dd}, J=12.6 \mathrm{~Hz}, J=4.0 \mathrm{~Hz}, 2 \mathrm{H}$, $\mathrm{H} H \mathrm{C}-\mathrm{CH}(\mathrm{O}-)-\mathrm{CHH}), 1.68$ (t, $J=12.0 \mathrm{~Hz}, 2 \mathrm{H}, H \mathrm{HC}-\mathrm{CHOCO}-$ $\mathrm{CH} H), 2.00(\mathrm{dd}, J=4.0 \mathrm{~Hz}, J=12.6 \mathrm{~Hz}, 2 \mathrm{H}, \mathrm{H} H \mathrm{C}-\mathrm{CHOCO}-$ $\mathrm{CHH}), 5.21$ (tt, $J=11.3 \mathrm{~Hz}, J=4.4 \mathrm{~Hz}, 1 \mathrm{H}, \mathrm{CH}-\mathrm{OC}(=\mathrm{O})), 6.52$ $(\mathrm{dd}, J=16.0 \mathrm{~Hz}, J=0.6 \mathrm{~Hz}, 1 \mathrm{H}, \mathrm{Ar}-\mathrm{CH}=\mathrm{C} H-\mathrm{COO}), 7.32$ (dd, $J$ $=16.0 \mathrm{~Hz}, J=0.6 \mathrm{~Hz}, 1 \mathrm{H}, \mathrm{Ar}-\mathrm{CH}=\mathrm{CH}-\mathrm{COO}) ;{ }^{13} \mathrm{C} \mathrm{NMR}(50$ $\left.\mathrm{MHz}, \delta, \mathrm{CDCl}_{3}, \mathrm{TMS}, \mathrm{PhNHNH}_{2}\right) 20.73\left(2 \times \mathrm{CH}_{3}\right), 32.01$ $\left(2 \times \mathrm{CH}_{3}\right), 44.02\left(2 \times \mathrm{CH}_{2}\right), 59.79\left(2 \times C\left(\mathrm{CH}_{3}\right)_{2}\right), 67.62(\mathrm{CH}-\mathrm{O}-)$, $122.89(\mathrm{CH}), 124.37(\mathrm{CH}), 128.54(\mathrm{C}), 128.84(\mathrm{CH}), 140.69$ (C), $141.92(\mathrm{CH}), 165.71(\mathrm{C}=\mathrm{O})$; IR $\left(\mathrm{cm}^{-1}, \mathrm{KBr}\right) 1705,1638$, $1519,1344,1177,850$.

\section{2,2,6,6-Tetramethyl-1-oxyl-4-piperidyl 3-E-(2- methyl-4-nitrophenyl)acrylate (5h)}

88.3\%; mp 109-112 ${ }^{\circ} \mathrm{C}$; MS (EI, $70 \mathrm{eV}, \mathrm{m} / \mathrm{z}$, int [\%]) 361 (15, $\mathrm{M}^{+}$), 347 (4), 331 (7), 191 (32), 190 (74, ArCH=CHCO), 174 (11), 161 (6), 160 (10), 154 (29), 144 (37), 140 (57), 139 (26), 124 (100), 116 (47), 115 (60), 109 (82), 98 (12), 95 (11), 89 (10), 82 (36), 81 (30), 69 (33), 68 (20), 67 (27), 58 (9), 57 (12), 56 (21), 55 (30), 43 (15), 42 (12), 41 (45); HRMS (EI, $70 \mathrm{eV,}$ $m / z$, int [\%]): calcd for $\mathrm{C}_{19} \mathrm{H}_{25} \mathrm{~N}_{2} \mathrm{O}_{5}: 361.1763$, found, 361.1761; ${ }^{1} \mathrm{H} \mathrm{NMR}\left(200 \mathrm{MHz}, \delta, \mathrm{CDCl}_{3}, \mathrm{TMS}, \mathrm{PhNHNH}_{2}\right.$ ) $1.26\left(\mathrm{~s}, 6 \mathrm{H},\left(\mathrm{CH}_{3}\right)\left(\mathrm{CH}_{3}\right) \mathrm{CN}(\mathrm{OH})-\mathrm{C}\left(\mathrm{CH}_{3}\right)\left(\mathrm{CH}_{3}\right)\right), 1.28(\mathrm{~s}, 6 \mathrm{H}$, $\left.\left(\mathrm{CH}_{3}\right)\left(\mathrm{CH}_{3}\right) \mathrm{CN}(\mathrm{OH}) \mathrm{C}\left(\mathrm{CH}_{3}\right)\left(\mathrm{CH}_{3}\right)\right), 1.74(\mathrm{t}, J=12.0 \mathrm{~Hz}, 2 \mathrm{H}$, $H \mathrm{HC}-\mathrm{CH}(\mathrm{O}-)-\mathrm{CH} H), 2.03$ (ddd, $J=11.2 \mathrm{~Hz}, J=4.4 \mathrm{~Hz}, J=1.4$ $\mathrm{Hz}, 2 \mathrm{H}, \mathrm{H} H \mathrm{C}-\mathrm{CH}(\mathrm{O}-)-\mathrm{CHH}), 2.51\left(\mathrm{ArCH}_{3}\right), 5.22(\mathrm{tt}, J=11.3$ $\mathrm{Hz}, J=4.3 \mathrm{~Hz}, 1 \mathrm{H}, \mathrm{CH}-\mathrm{OC}(=\mathrm{O})), 6.42(\mathrm{~d}, J=15.9 \mathrm{~Hz}, 1 \mathrm{H}$, $\mathrm{Ar}-\mathrm{CH}=\mathrm{C} H$-COO $), 7.90(\mathrm{~d}, J=15.9 \mathrm{~Hz}, 1 \mathrm{H}, \mathrm{Ar}-\mathrm{CH}=\mathrm{CH}-$ $\mathrm{COO}) ;{ }^{13} \mathrm{C} \mathrm{NMR}\left(50 \mathrm{MHz}, \delta, \mathrm{CDCl}_{3}, \mathrm{TMS}, \mathrm{PhNHNH}_{2}\right) 20.10$ $\left(\mathrm{ArCH}_{3}\right), 20.90\left(2 \times \mathrm{CH}_{3}\right), 31.66\left(2 \times \mathrm{CH}_{3}\right), 43.81\left(2 \times \mathrm{CH}_{2}\right)$, 
$60.32\left(2 \times C\left(\mathrm{CH}_{3}\right)_{2}\right), 67.47(\mathrm{CH}-\mathrm{O}-), 121.62(\mathrm{CH}), 123.52(\mathrm{CH})$, $125.72(\mathrm{CH}), 127.52(\mathrm{CH}), 128.95(\mathrm{C}), 139.22(\mathrm{C}), 139.93(\mathrm{C})$, 140.21 (CH), $165.86(\mathrm{C}=\mathrm{O})$; IR $\left(\mathrm{cm}^{-1}, \mathrm{KBr}\right) 1715,1519,1347$, 1170 .

\section{2,2,6,6-Tetramethyl-1-oxyl-4-piperidyl 3-E-(2- chloro-4-nitrophenyl)acrylate (5i)}

56.2\%; mp 107-110 ${ }^{\circ} \mathrm{C}$; MS (EI, $70 \mathrm{eV}, \mathrm{m} / \mathrm{z}$, int [\%]) 383 (1), $382(1), 381\left(2, \mathrm{M}^{+}\right), 212(14), 211$ (9), 210 (41, $\mathrm{ArCH}=\mathrm{CHCO}), 194$ (4), 192 (8), 166 (4), 164 (11), 154 (15), 140 (17), 139 (14), 138 (9), 136 (23), 124 (89), 109 (100), 101 (13), 100 (19), 99 (7), 98 (9), 95 (8), 89 (13), 82 (19), 81 (22), 75 (12), 74 (12), 69 (19), 68 (13), 67 (23), 57 (14), 56 (17), 55 (28), 43 (18), 41 (58); MS (ESI, $m / z$, int [\%]): $404(15, \mathrm{M}+$ $\mathrm{Na}), 249$ (100), 242 (15); HRMS (ESI): calcd for $\mathrm{C}_{18} \mathrm{H}_{22} \mathrm{~N}_{2} \mathrm{O}_{5} \mathrm{ClNa}$ : 404.1115, found, 404.1131; ${ }^{1} \mathrm{H}$ NMR (200 $\left.\mathrm{MHz}, \delta, \mathrm{CDCl}_{3}, \mathrm{TMS}, \mathrm{PhNHNH}_{2}\right) 1.25,1.27,1.29(3 \mathrm{~s}, 12 \mathrm{H}$, $\left.4 \times \mathrm{CH}_{3}\right), 1.75(\mathrm{~m}, 2 \mathrm{H}, H \mathrm{HC}-\mathrm{CH}(\mathrm{O}-)-\mathrm{CH} H), 2.01$ (m, 2H, HHC$\mathrm{CH}(\mathrm{O}-)-\mathrm{CHH}), 5.22(\mathrm{tt}, J=11.3 \mathrm{~Hz}, J=4.3 \mathrm{~Hz}, 1 \mathrm{H}$, $\mathrm{CH}-\mathrm{OC}(=\mathrm{O})), 6.51(\mathrm{~d}, J=16.0 \mathrm{~Hz}, 1 \mathrm{H}, \mathrm{Ar}-\mathrm{CH}=\mathrm{CH}-\mathrm{COO})$, $8.03(\mathrm{~d}, J=16.0 \mathrm{~Hz}, 1 \mathrm{H}, \mathrm{Ar}-\mathrm{C} H=\mathrm{CH}-\mathrm{COO}) ;{ }^{13} \mathrm{C}$ NMR $(50$ $\left.\mathrm{MHz}, \delta, \mathrm{CDCl}_{3}, \mathrm{TMS}, \mathrm{PhNHNH}_{2}\right) 20.88\left(2 \times \mathrm{CH}_{3}\right), 31.67$ $\left(2 \times \mathrm{CH}_{3}\right), 43.75\left(2 \times \mathrm{CH}_{2}\right), 60.33\left(2 \times C\left(\mathrm{CH}_{3}\right)_{2}\right), 67.72(\mathrm{CH}-\mathrm{O}-)$, $122.22(\mathrm{CH}), 125.02(\mathrm{CH}), 125.58(\mathrm{CH}), 128.53(\mathrm{C}), 128.87$ (C), $128.90(\mathrm{CH}), 138.45(\mathrm{CH}), 139.10(\mathrm{C}), 165.31(\mathrm{C}=\mathrm{O})$; IR $\left(\mathrm{cm}^{-1}, \mathrm{KBr}\right)$ 2976, 1712, 1523, 1346, 1330, 1194, 1180, 990.

\section{Supporting Information}

\section{Supporting Information File 1 \\ EIMS, ESIMS, ${ }^{1} \mathrm{H}$ NMR, ${ }^{13} \mathrm{C}$ NMR, and IR spectra of $\mathbf{3}$ and $\mathbf{5 a}-\mathbf{5 i}$. \\ [http://www.beilstein-journals.org/bjoc/content/ supplementary/1860-5397-11-130-S1.pdf]}

\section{Acknowledgements}

The work was supported by the Polish Ministry of Science and Higher Education, 429/E-142/S/2012.

\section{References}

1. Zakrzewski, J.; Krawczyk, M. Heterocycl. Commun. 2014, 20, 89-91. doi:10.1515/hc-2013-0169

2. Sova, M. Mini-Rev. Med. Chem. 2012, 12, 749-767. doi:10.2174/138955712801264792

3. De, P.; Bedos-Belval, F.; Vanucci-Bacqué, C.; Baltas, M. Curr. Org. Chem. 2012, 16, 747-768. doi:10.2174/138527212799958020

4. De, P.; Baltas, M.; Bedos-Belval, F. Curr. Med. Chem. 2011, 18, 1672-1703. doi:10.2174/092986711795471347
5. Clifford, M. N. J. Sci. Food Agric. 2000, 80, 1033-1043. doi:10.1002/(SICI)1097-0010(20000515)80:7<1033::AID-JSFA595>3.0 .CO;2-T

6. Clifford, M. N. J. Sci. Food Agric. 1999, 79, 362-372. doi:10.1002/(SICI)1097-0010(19990301)79:3<362::AID-JSFA256>3.0 CO;2-D

7. Sharma, P. J. Chem. Pharm. Res. 2011, 3, 403-423.

8. Narasimhan, B.; Belsare, D.; Pharande, D.; Mourya, V.; Dhake, A. Eur. J. Med. Chem. 2004, 39, 827-834. doi:10.1016/j.ejmech.2004.06.013 And references cited therein.

9. Sambandan, D. R.; Ratner, D. J. Am. Acad. Dermatol. 2011, 64, 748-758. doi:10.1016/j.jaad.2010.01.005

10. Bhatia, S. P.; Cocchiara, J.; Wellington, G. A.; Lalko, J.; Letizia, C. S.; Api, A. M. Food Chem. Toxicol. 2011, 49 (Suppl. 2), S252-S255. doi:10.1016/j.fct.2011.07.051

11. Belsito, D.; Bickers, D.; Bruze, M.; Calow, P.; Dagli, M.; Fryer, A. D.; Greim, H.; Miyachi, Y.; Saurat, J. H.; Sipes, I. G. Food Chem. Toxicol. 2011, 49 (Suppl. 2), S256-S267. doi:10.1016/j.fct.2011.07.053

12. Waghmare, S. R.; Gaikwad, H. K. J. Chem. Pharm. Res. 2012, 4 , 2415-2421.

13. Wall, V. M.; Eisenstadt, A.; Ager, D. J.; Laneman, S. A. Platinum Met. Rev. 1999, 43, 138-145.

14. Sheng, S.-R.; Luo, Q.-Y.; Huang, P.-G.; Guo, L.; Wang, Q.-Y.; Pei, X.-L. J. Chem. Res. 2006, 24-26. doi:10.3184/030823406776331070

15. Liu, S.; Fukuyama, T.; Sato, M.; Ryu, I. Org. Process Res. Dev. 2004, 8, 477-481. doi:10.1021/op034200h

16. Mingji, D.; Liang, B.; Wang, C.; You, Z.; Xiang, J.; Dong, G.; Chen, J.; Yang, Z. Adv. Synth. Catal. 2004, 346, 1669-1673. doi:10.1002/adsc.200404165

17. Bumagin, N. A.; More, P. G.; Beletskaya, I. P. J. Organomet. Chem. 1989, 371, 397-401. doi:10.1016/0022-328X(89)85235-0

18. Smith, M. R.; Kim, J. Y.; Ciufolini, M. A. Tetrahedron Lett. 2013, 54, 2042-2045. doi:10.1016/j.tetlet.2013.01.077

19. Pastre, J. C.; Correia, C. R. D. Adv. Synth. Catal. 2009, 351, 1217-1223. doi:10.1002/adsc. 200900032

20. Sakakibara, T.; Nishimura, S.; Kimura, K.; Minato, I.; Odaira, Y. J. Org. Chem. 1970, 35, 3884-3887. doi:10.1021/jo00836a066

21. Zakrzewski, J. Beilstein J. Org. Chem. 2012, 8, 1515-1522. doi:10.3762/bjoc.8.171

22. Kálai, T.; Borza, E.; Antus, C.; Radnai, B.; Gulyás-Fekete, G.; Fehér, A.; Sümegi, B.; Hideg, K. Bioorg. Med. Chem. 2011, 19 7311-7317. doi:10.1016/j.bmc.2011.10.066

23. Kálai, T.; Bognár, B.; Jekő, J.; Hideg, K. Synthesis 2006, 2573-2579. doi:10.1055/s-2006-942439

24. Keddie, D. J.; Johnson, T. E.; Arnold, D. P.; Bottle, S. E. Org. Biomol. Chem. 2005, 3, 2593-2598. doi:10.1039/b504354a

25. Keddie, D. J. The Synthesis of Novel Profluorescent Nitroxide Probes. Ph.D. Thesis, Queensland University of Technology, Austraöia, 2008.

26. Fairfull-Smith, K. E.; Bottle, S. E. Eur. J. Org. Chem. 2008, 5391-5400. doi:10.1002/ejoc.200800597

27. Kálai, T.; Balog, M.; Jekő, J.; Hubbell, W. L.; Hideg, K. Synthesis 2002, 2365-2372. doi:10.1055/s-2002-35240

28. Stroh, C.; Mayor, M.; von Hänisch, C. Eur. J. Org. Chem. 2005, 3697-3703. doi:10.1002/ejoc.200500116

29. Kálai, T.; Jekő, J.; Berente, Z.; Hideg, K. Synthesis 2006, 439-446. doi:10.1055/s-2006-926279

30. Keddie, D. J.; Fairfull-Smith, K. E.; Bottle, S. E. Org. Biomol. Chem. 2008, 6, 3135-3143. doi:10.1039/B806963H 
31. Miura, Y.; Ushitani, Y. Macromolecules 1993, 26, 7079-7082. doi:10.1021/ma00077a056

32. Romero, F. M.; Ziessel, R. Tetrahedron Lett. 1999, 40, 1895-1898. doi:10.1016/S0040-4039(99)00069-6

33. Neiman, M. B.; Rozantsev, E. G.; Mamedova, Yu. G. Nature 1962, 196, 472-474. doi:10.1038/196472a0

34. Hyslop, D. K.; Parent, J.; Scott, J. S. Macromolecules 2012, 45 , 8147-8154. doi:10.1021/ma3016135

35. Appelt, M.; Schmidt-Naake, G. Macromol. Mater. Eng. 2004, 289, 245-253. doi:10.1002/mame.200300255

36. Rozantsev, E. G.; Suskina, V. J. Russ. Chem. Bull. 1968, 17, 1997-1999. Izv.. Akad.. Nauk. SSSR, S. Kh. 1968, 2106-2109.

37. Dieck, H. A.; Heck, R. F. J. Am. Chem. Soc. 1974, 96, 1133-1136. doi:10.1021/ja00811a029

38. Coulson, D. R.; Satek, L. C.; Grim, S. O. Inorg. Synth. 1972, 13, 121-124. doi:10.1002/9780470132449.ch23

39. Shezad, N.; Oakes, R. S.; Clifford, A. A.; Rayner, C. M. Tetrahedron Lett. 1999, 40, 2221-2224. doi:10.1016/S0040-4039(99)00176-8

40. Reaxa Ltd, Aldrich; Pd EnCat ${ }^{\mathrm{TM}}$, Experimental Guide.

41. Reaxa Ltd; Pd(II) EnCat ${ }^{\mathrm{TM}}$ Experimental User Guide. May 2006.

42. Mendes Da Silva, J. F.; Yepes Perez, A. F.; Pinto de Almeida, N. RSC Adv. 2014, 4, 28148-28155. doi:10.1039/C4RA03586K

43. Barros, J. C.; Yaunner, R. S.; de Souza, A. L. F.; da Silva, J. F. M.; Antunes, O. A. C. Appl. Organomet. Chem. 2011, 25, 820-823. doi:10.1002/aoc.1845

44. Broadwater, S. J.; McQuade, D. T. J. Org. Chem. 2006, 71, 2131-2134. doi:10.1021/jo0517904

45. Lee, C. K. Y.; Holmes, A. B.; Ley, S. V.; McConvey, I. F.; Al-Duri, B.; Leeke, G. A.; Santos, R. C. D.; Seville, J. P. K. Chem. Commun. 2005, 2175-2177. doi:10.1039/b418669a

46. Tandukar, S.; Sen, A. J. Mol. Catal. A: Chem. 2007, 268, 112-119. doi:10.1016/j.molcata.2006.12.003

47. Leikoski, T.; Wrigstedt, P.; Helminen, J.; Matikainen, J.; Sipilä, J.; Yli-Kauhaluoma, J. Tetrahedron 2013, 69, 839-843. doi:10.1016/j.tet.2012.10.092

48. Smith, M. R.; Jang, Y. I.; Kim, J. Y.; Ciufolini, M. A. Tetrahedron 2013, 69, 10139-10151. doi:10.1016/j.tet.2013.09.019

49. Lee, T. D.; Keana, J. F. W. J. Org. Chem. 1975, 40, 3145-3147. doi:10.1021/j000909a033

50. López-Peña, H. A.; Hernández-Muñoz, L. S.; Frontana-Uribe, B. A.; González, F. J.; González, I.; Frontana, C.; Cardoso, J. J. Phys. Chem. B 2012, 116, 5542-5550. doi:10.1021/jp301207v

51. Li, Y.; Lei, X.; Li, X.; Lawler, R. G.; Murata, Y.; Komatsu, K.; Turro, N. J. Chem. Commun. 2011, 47, 12527-12529. doi:10.1039/c1cc15149e

52. Li, Y.; Lei, X.; Lawler, R. G.; Murata, Y.; Komatsu, K.; Turro, N. J. Org. Lett. 2012, 14, 3822-3825. doi:10.1021/ol3013155

53. Morrison, A.; Davies, A. P. Org. Mass Spectrom. 1970, 3, 353-366. doi:10.1002/oms.1210030310

54. Kaliská, V.; Toma, Š.; Leško, J. Collect. Czech. Chem. Commun. 1987, 52, 2266-2273. doi:10.1135/cccc19872266

\section{License and Terms}

This is an Open Access article under the terms of the Creative Commons Attribution License

(http://creativecommons.org/licenses/by/2.0), which permits unrestricted use, distribution, and reproduction in any medium, provided the original work is properly cited.

The license is subject to the Beilstein Journal of Organic Chemistry terms and conditions:

(http://www.beilstein-journals.org/bjoc)

The definitive version of this article is the electronic one which can be found at:

doi:10.3762/bjoc. 11.130 